\title{
BMJ Open Bleeding risk in patients using oral anticoagulants submitted to surgical procedures in dentistry: a systematic review protocol
}

\author{
Rogério Heládio Lopes Motta, ${ }^{1}$ Cristiane de Cássia Bergamaschi, ${ }^{2}$ \\ Natalia Karol de Andrade, ${ }^{1}$ Caio Chaves Guimaraes, ${ }^{1}$ Juliana Cama Ramacciato, ${ }^{1}$ \\ Jimmy de Oliveira Araújo, ${ }^{1}$ Luciane Cruz Lopes ${ }^{2}$
}

To cite: Motta RHL, Bergamaschi CdC, de Andrade NK, et al. Bleeding risk in patients using oral anticoagulants submitted to surgical procedures in dentistry: a systematic review protocol. BMJ Open 2017;7:e019161. doi:10.1136/ bmjopen-2017-019161

- Prepublication history for this paper is available online. To view these files, please visit the journal online (http://dx.doi. org/10.1136/bmjopen-2017019161).

Received 16 August 2017 Revised 2 October 2017 Accepted 4 October 2017

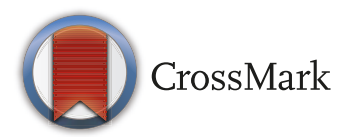

${ }^{1}$ Pharmacology, Anesthesiology and Therapeutics, São Leopoldo Mandic Dental School and Research Center, Campinas, São Paulo, Brazil

${ }^{2}$ Department of Pharmaceutical Sciences Graduate Program,

University of Sorocaba,

Sorocaba, São Paulo, Brazil

Correspondence to Dr Cristiane de

Cássia Bergamaschi; cristiane.motta@prof.uniso.br

\section{ABSTRACT}

Introduction The management of patients undergoing oral surgical procedures using anticoagulants raises concerns regarding the risk of bleeding. Bleeding rates in those patients during or after oral surgical procedures are uncertain. The aim of this study will be to determine the bleeding rate during and after oral surgeries in patients using anticoagulants.

Methods and analysis A systematic review will be conducted and if appropriate, a meta-analysis of randomised clinical trials evaluating the bleeding risk during and after oral surgical procedures in patients using anticoagulants were selected. The literature search will be conducted using electronic databases, such as the Cochrane Central Register of Controlled Trials, MEDLINE (via Ovid), Embase (via Ovid), Cumulative Index to Nursing and Allied Health Literature (via Ovid), LILACS (SciELO) and CAPES database, without restriction of languages or date of publication. The primary outcome will be the occurrence of local bleeding rate during and after oral surgical procedures, and as secondary outcome, the following complications will be considered: implant failure, healing problems and infections. Groups of two independent reviewers will select the titles and abstracts for full-text eligibility. For eligible studies, the same reviewers will perform data extraction, bias risk assessment and determination of the overall quality of evidence for each of the outcomes using the Grading of Recommendations Assessment, Development and Evaluation classification system. Meta-analysis and subgroup analyses will be conducted, to all outcomes, if appropriate.

Ethics and dissemination The systematic review will be published in a peer-reviewed journal, and brief reports of the review's findings will be released directly to the intended audience. The results will help dentists in the decisionmaking process to minimise the risk of bleeding in patients using anticoagulants in their clinical practice. PROSPERO registration number CRD42017056986.

\section{INTRODUCTION}

The use of oral anticoagulants has been indicated for the prevention of thromboembolism in patients suffering from heart diseases such as ischaemic cardiac disease, atrial
Strengths and limitations of this study

- Bleeding is a negative outcome in dentistry that limits several surgical interventions. Bleeding risk rate in users of oral anticoagulants may contribute to minimise bleeding risk for these patients.

- The use of Grading of Recommendations Assessment, Development and Evaluation will evaluate the strength and quality of the evidence body concerning the estimate of the effect for each outcome, including independent analysis of the risk bias, precision, consistency, publication bias and indirect evidence.

- The method of this review includes explicit eligibility criteria, comprehensive and extensive search in database, independent and paired evaluation and study eligibility. Rates for body of evidence of the studies included in the review will be estimated.

- The quality of the primary studies to be included in this review may be a limiting factor if there is heterogeneity in study design and outcome measurements.

fibrillation and patients with prosthetic heart valves, among others. ${ }^{12}$

Therefore, some diseases such as venous thromboembolism, acute arterial occlusion, valvulopathy with subsequent placement of the valve prosthesis and atrial fibrillation have indication for the use of anticoagulants. The use of these drugs is due to the changes in the haemostatic balance between clotting and blood anticoagulation, and any other more significant change in this balance may cause risk of haemorrhage or thromboembolism. ${ }^{3}$

Haemostatic balance keeps constant equilibrium between the creation and destruction of thrombus. This equilibrium is kept by a complex interaction among platelets and vascular endothelium, the coagulation cascade and the fibrinolytic 
system. Blood clotting is one step of the haemostatic mechanism working in the formation of a solid mass composed by a fibrin net that contains blood elements such as white cell count, red blood cells and platelets. $^{45}$

Anticoagulants may be classified according to its route of administration, for example, oral (warfarin, apixaban, rivaroxaban, dabigatran, argatroban, ximelagatran, among others) and parenteral (high molecular weight heparin, dalteparin, enoxaparin, among others). The increase in number of these drug users has enabled the chances dentist will treat patients undergoing anticoagulant therapies. ${ }^{6} 7$

Some studies have demonstrated that exodontias, when conducted in patients with desirable international normalised ratio (INR), may be safely conducted and without warfarin and antiplatelet drugs interruption, ${ }^{8-17}$ while another study demonstrated a $3.24 \%$ difference after exodontias between patients using warfarin and others not using the drug. ${ }^{18}$

Some studies of systematic reviews evaluated the bleeding risk in patients using anticoagulants and submitted to oral interventions. ${ }^{3} 1416$ 19-22 However, some topics raised deserve special attention: the need to update the literature and/or of risk of bias assessment ${ }^{314} 1522$; the absence of findings regarding the quality of the evidence ${ }^{314} 1^{19-21}$; the fact that some studies mixed different methodological designs in their analyses (randomised clinical trial (RCT), controlled clinical trial, guidelines, cohort and case series) ${ }^{3} 1920$; and the fact that some studies did not restrict INR values. ${ }^{21}$ Therefore, considering these topics mentioned and the importance on the subject, new studies have been recommended.

These protocols have been researched in dentistry in order to guarantee a treatment that prevents the occurrence of bleedings and does not expose the patient to risk of thromboembolism. Therefore, this systematic review aims to determine the bleeding risk during and after oral interventions in patients using anticoagulants to help professionals to make decisions in clinical practice in order to minimise the risks and have adequate management in the conduction of these interventions.

\section{METHODS AND ANALYSES}

A systematic review will be conducted according to the recommendations described in Cochrane Handbook for Intervention Reviews. ${ }^{23}{ }^{24}$ Evaluation will be done according to the items from Preferred Reporting Items for Systematic Reviews and Meta-Analyses statement. ${ }^{23} 25$

\section{Protocol and registration}

Review protocol was registered with the International Prospective Register of Systematic Reviews (protocol CRD42017056986) (http://www.crd.york.ac.uk/PROSPERO).

\section{Eligibility criteria}

\section{Inclusion criteria}

- Participants: adult patients of both gender (over 18 years old) in need of oral surgery such as exodontias and implant placement.

- Intervention: the studies will include a group of patients anticoagulated with VKA or new anticoagulants and a control group (placebo).

- Study design: RCT.

\section{Exclusion criteria}

- Patients: studies with over $20 \%$ of patients using two or more antithrombotic agents and studies with population that is clearly not representative, that is, with different bleeding risks (eg, patients with recent episode of cerebrovascular accident, patients who had ablation procedures recently and so on).

- Interventions: studies that considered INR out of the interval 2.0 and $3.0 .^{26}$

\section{Evaluated outcomes}

Studies shall report at least one of the following outcomes: postsurgical or transoperative bleeding measured at least 48 hours after oral surgical intervention. The definition of bleeding will be accepted as described in each study. Studies may also report oral complications (infections, implant failures and healing problems at the surgical site), considered as secondary outcomes.

\section{Search methods for identification of studies Electronic database search}

The following electronic database will be searched: Cochrane Central Register of Controlled Trials part of The Cochrane Library; MEDLINE (via Ovid); Embase (via Ovid); Cumulative Index to Nursing and Allied Health Literature (via Ovid) and BVS (Digital Library about Health) without restriction of languages or date of publication.

\section{Search strategy}

Terms that individually describe bleeding risks and oral surgical procedures will be combined. Search strategy will be adapted for each database. MEDLINE search strategy is provided in box.

\section{Other resources for reference search}

Two reviewers will review the reference list of every eligible study or citations found in secondary studies in order to verify possibly eligible studies. When necessary, the main authors of the study will be contacted for further information.

\section{Eligibility determination}

Four reviewers (RHLM, CdCB, LCL and NKdA), working in pairs, will independently screen potentially relevant citations and abstracts and will apply the selection criteria. Full texts of all articles will be obtained in case either reviewer feels they might be eligible. Two reviewers will independently assess the eligibility of each full-text 


\section{Box Search strategy of database: Ovid MEDLINE (1950 to present)}

1. Oral surgery.mp. or exp oral surgery/

2. Maxillofacial Surgery.mp.

3. Surgery, Maxillofacial.mp.

4. Oral Surgery.mp.

5. Exodontics.mp

6. Dentistry, Operative.mp.

7. Oral Surgical Procedures.mp.

8. Dental Implants.mp or exp Dental Implant/

9. Implants, Dental.mp.

10. Dental Implant.mp.

11. Implant, Dental.mp.

12. Dental Prostheses, Surgical.mp.

13. Dental Prosthesis, Surgical.mp.

14. Surgical Dental Prostheses.mp.

15. Surgical Dental Prosthesis.mp.

16. Prosthesis, Surgical Dental.mp.

17. Prosthesis, Surgical Dental.mp.

18. 1 OR 2 OR 3 OR 4 OR 5 OR 6 OR 7 OR 8 OR 9 OR 10 OR 11 OR 12 OR 13 OR 14 OR 15 OR 16 OR 17

19. exp Hemorrhage/or hemorrhage.mp.

20. bleeding\$.mp.

21. bleed $^{*} . \mathrm{mp}$.

22. microbleed $^{*} \cdot \mathrm{mp}$.

23. exp Risk/

24. $\exp$ Risk Factors/

25. exp Risk Assessment/

26. risk*.mp.

27. 19 OR 20 OR 21 OR 22 OR 23 OR 24 OR 25 OR 26

28. 18 AND 27

29. exp Coumarins/

30. (coumarin $\$$ or chromonar or coumestrol or esculin or isocoumarin $\$$ or psoralens or pyranocoumarins or umbelliferones).mp.

31. warfarin.mp. or Warfarin/or warfant.mp.

32. (coumadine or warfant or coumadin or marevan or aldocumar or tedicumar).mp.

33. Acenocoumarol/or acenocoumarol*.mp.

34. (Pradaxa or Dabigatran).mp.

35. (Rivaroxaban or Xarelto).mp.

36. (vitamin $\mathrm{k}$ adj3 antagonis*).mp.

37. vitamin $\mathrm{k} / \mathrm{ai}$

38. (vk adj2 antagonis*).mp.

39. (endosaban or apixaban).mp.

40. BAY 59-7939.mp.

41. (BMS-562247 or edoxaban or DU-176b or betrixaban).mp.

42. YM150.mp.

43. TAK-442.mp.

44. LY517717.mp.

45. PD0348292.mp.

46. (VKA or VKAs).mp.

47. (NOACs or noac).mp.

48. (DOACs or doac).mp.

49. ((new or novel or direct) adj4 (oral anticoag* or oral anti coag*)).mp.

50. ((novel or new) adj2 (anticoag: or anti coag:)).mp.

51. exp Heparin/

52. (LMWH or heparin or nadroparin or fraxiparin or enoxaparin or clexane or lovenox or dalteparin or fragmin or ardeparin or normiflo or tinzaparin or logiparin or innohep or certoparin or sandoparin or reviparin or clivarin or danaproid or orgaran or bemiparin or hibor, badyket, semuloparin, parnaparin, fluxum).tw.

53. exp Coumarins/

54. (warfarin or coumadin or acenocoumarol or phenprocoumon or 4-hydroxycoumarins or oral anticoagulant or vitamin K antagonist or VKA).tw.

55. (fondaparinux or arixtra).tw.

56. (ximelagatran or exanta).tw.

57. (pradaxa or dabigatran or rivaroxaban or xarelto or apixaban or eliquis or edoxaban or lixiana or betrixaban or edoxaban or otamixaban).tw. 
Box Search strategy of database: Ovid MEDLINE (1950 to present) Continued

58. 29 or 30 or 31 or 32 or 33 or 34 or 35 or 36 or 37 or 39 or 40 or 41 or 42 or 43 or 44 or 45 or 46 or 47 or 48 or 49 or 50 or 51 or 52 or 53 or 54 or 55 or 56 or 57

59. 28 AND 58

article and resolve disagreements by consensus. In case of duplicate publication, the article with the more complete data will be used.

Kappa statistics will be used to measure agreement between the examiners. Values of kappa between 0.40 and 0.59 have been considered to reflect fair agreement, values between 0.60 and 0.8 reflect good agreement and values that are 0.75 or more reflect excellent agreement. ${ }^{27}$

\section{Data extraction}

Four reviewers (RHLM, CdCB, LCL and NKdA), working in pairs, will independently extract the data and will record information regarding patients, methods, interventions, outcomes and missing outcome data using standardised and pretested data extraction forms with instructions. Two reviewers, in pairs, and independently, will be calibrated in the extraction of at least three articles, and then shall enter in a consensus. This procedure shall occur until the reviewers are able to extract data. Authors of the study will be contacted to solve any doubts. Disagreements will be resolved by consensus concerning any unresolved issues referred to another reviewer.

For the articles that are published only as abstracts or articles with important missing information, their authors shall be contacted in order to obtain complete information regarding the missing methods and results.

\section{Bias risk}

A modified version of Cochrane collaboration will be used for bias risk. ${ }^{22} 28$ The reviewers will independently assess the risk of bias for each randomised trial, according to the following criteria: random sequence; allocation concealment; blinding of the patient, healthcare professionals, outcome assessors, data collectors and data analysts; incomplete outcome data; selective outcome reporting; and major baseline imbalance. Reviewers will assign response options of 'definitely yes', 'probably yes', 'probably no' and 'definitely no' for each of the domains, with 'definitely yes' and 'probably yes' ultimately being assigned a low risk of bias and 'definitely no' and 'probably no' a high risk of bias. ${ }^{29}$ Reviewers will resolve disagreements by consensus and one arbitrator (LCL) will settle unresolved disagreements.

Possible explanations for heterogeneity will include the following: doses (higher vs lower) with an expected larger effect with higher doses, duration of the treatment (longer vs shorter) with an expected larger effect with longer duration of the treatment and the risk of bias, with an expected larger effect in trials at high or unclear risk of bias versus trials at low risk of bias.

\section{Confidence in pooled estimates of effects}

The quality of evidence from randomised trials for each of the outcomes will also be independently rated by using Grading of Recommendations Assessment, Development and Evaluation (GRADE) approach. ${ }^{30}{ }^{31}$ In the GRADE approach, randomised trials begin as high-quality evidence but may be rated down by one or more of five categories of limitations: risk of bias, inconsistency, indirectness, imprecision and reporting bias.

Considering that bleeding rates are different among the studies, some hypothesis will be established and considered as heterogeneity: (A) age distribution (old age, higher incidence of bleeding); (B) gender distribution (women - higher incidence); (C) ethnic groups (geographic area); (D) period of time for surgical procedure (longer periods, higher risk of bleeding); (E) type of surgical procedure (more invasive, more risk of bleeding); (F) use of local anaesthetic without vasoconstrictor (without vasoconstrictor, higher risk of bleeding); (G) proportion of patients using only one anticoagulant drug (higher risk with patients using more than one anticoagulant drugs); (H) proportion of present or remote bleeding event (higher risk in patients with present event); and (I) alcohol/drug abuse (higher risk in this kind of patient).

Heterogeneity associated with pooled effect estimates will be assessed with the use of a $\mathrm{X}^{2}$ test and the $\mathrm{I}^{2}$ statistic. ${ }^{32}$ The following heterogeneity will be considered: $0 \%-40 \%$ (no important heterogeneity); 30\%-60\% (moderate heterogeneity); 50\%-90\% (substantial heterogeneity); and 75\%-100\% (considerable heterogeneity).

\section{Data synthesis}

Analyses will be conducted for each coagulant and for each outcome of interest. Confidence will be determined through estimates for each body of evidence, and analysis will be conducted for the body of evidence that ensures greater confidence.

Hypotheses will be examined regarding which information will be documented in at least 10 studies for independent continuous variables or in at least five studies for independent categorical variables.

Combined analysis will estimate the bleeding rate for 100 patients-years exposed to coagulants, severe haemorrhage and its subcategories. Variance estimates will be used based on CIs, when possible, or the total number of patient-years of exposure. If rates, CI and total exposure are not available, the exposure of the average years of follow-up will be estimated. 
When the total number of bleeding or patients who had haemorrhage are available but bleeding rates are not, bleeding rates will be estimated based on the total number or bleeding proportion and the average period of follow-up. For cases in which studies do not report any event, the 0.5 correction of continuity will be used. Estimates will be grouped in $\log$ scale units with the use of DerSimonian and Laird models of random effects considered by the inverse variation approach and later converted to the rates in natural units.

Meta-analyses will be conducted using Comprehensive Meta-Analysis STATA software (V.10.1). Random effects meta-analyse ${ }^{27}$ will be used, which are conservative in the way within-studies and between-studies differences are considered in calculating the error term used in the analysis. For trials that report dichotomous outcomes, the pooled relative risk with associated $95 \%$ CI will be calculated.

For continuous data, weighted mean difference (WMD) will be used and its $95 \% \mathrm{CI}$ as effect measure. Once WMD has been calculated, this value will be contextualised by noting, when available, the corresponding anchor-based minimally important difference (MID), the smallest change in instrument score that patients perceive is important.

If studies reported the same construct using different measurement instruments, standardised mean difference (SMD) will be calculated as sensitivity analysis. The SMD expresses the intervention effect in SD units, rather than the original units of measurement, with the value of an SMD depending on the size of the effect (the difference between means) and the SD of the outcomes (the inherent variability among participants). For outcome measures that have an established anchor-based MID, this measure will be used to convert the SMD into an OR and risk difference. ${ }^{33}$

To facilitate the interpretation of the effects of continuous outcomes, the MID will be substituted, when MID is available for different scales, by the SD (denominator) in the SMD equation, which will result in more readily interpretable MID units instead of SD units. ${ }^{34}$ If an estimate of the MID is not available, a statistical approach developed by Suissa ${ }^{35}$ will be used to provide a summary estimate of the proportion of patients who benefit from treatment across all studies. Statistical approaches to enhance the interpretability of results of continuous outcomes outlined in this paragraph will use methods cited as well as those described by Thorlund $e t a l .{ }^{36}$ Funnel plots will be created to explore possible publication bias when at least 10 studies have contributed to a pooled analysis.

Combined analysis will be tested by Z-statistic, and heterogeneity measured by Q-statistic, among the studies analysed by $\chi^{2}$. When heterogeneity is present, a component of variance, due to the interstudy variance, will be incorporated in the calculation of the CI for the estimate. Studies not including any of the above data will not be included in the grouped estimate; for such studies, bleeding rates will be descriptively summarised.

Recently developed approaches will be used to address missing participant data for dichotomous outcomes ${ }^{29}$ and continuous outcomes. ${ }^{37}$ These approaches will only be applied to outcomes that meet the following criteria: present significant treatment effect and report sufficient missing participant data to potentially introduce clinically important bias. ${ }^{38}$ Thresholds for important missing participant data will be determined on an outcome-byoutcome basis.

Meta-analysis and subgroup analyses will be conducted, to all outcomes, if appropriate; however, if the meta-analysis is not appropriate due to excessive heterogeneity of population, intervention, comparator, outcome or methodology, summary tables will be made and narrative synthesis will be provided. ${ }^{39}$

\section{Summarising evidence}

Results in evidence profiles will be presented as recommended by the GRADE Working Group. ${ }^{40}$ Evidence profiles provide succinct, easily digestible presentations of quality of evidence and magnitude of effects. Our evidence profiles will be constructed with the help of a software program GRADEpro (http://ims.cochrane.org/gradepro) to include the following seven elements: (1) a list of up to seven important outcomes, both desirable and undesirable; (2) a measure of the typical burden of these outcomes (eg, control group and estimated risk); (3) a measure of the difference between risks with and without intervention; (4) the relative magnitude of effect; (5) numbers of participants and studies addressing these outcomes, as well as follow-up time; (6) a rating of the overall confidence in the estimate of effect for each outcome; and (7) comments, which will include the MID, if available.

\section{DISCUSSION}

This review will assess the possible evidence about the bleeding risk during and after oral surgical procedures in patients using anticoagulants in order to consistently and completely provide evidence estimates using GRADE approach. ${ }^{41}$ The results from this systematic review will help dentists make decisions in the clinical practice that will minimise the risks of haemorrhages in patients using anticoagulants in dental practice.

The data compiled about the bleeding risks in patients using anticoagulants will advise these professionals about the efficacy and safety of interventions and consequently facilitate clinical decisions. This study may also identify areas of interest for further investigations.

\section{ETHICS AND DISSEMINATION}

The submission of this study to the ethics committee is not necessary due to the fact that it is a systematic review. The systematic review will be published in a peer-reviewed journal and presented in conferences or congresses.

The evidence of this study will allow dentists to know about the efficacy and safety of anticoagulants in the decision-making process in the clinical practice in order to minimise the risk of haemorrhage in patients using these 
drugs in dental practice. Updates of this study should be conducted to inform and orient clinical practice.

Contributors RHLM is the principal investigator and led the writing of the manuscript. LCL and CdCB are the project managers and coinvestigators and contributed to the writing and revision of the manuscript. NKdA, CCG, JCR and JdOA are coinvestigators and contributed to the writing and revision of the manuscript. All authors read and approved the final manuscript.

Competing interests None declared.

Provenance and peer review Not commissioned; externally peer reviewed.

Open Access This is an Open Access article distributed in accordance with the Creative Commons Attribution Non Commercial (CC BY-NC 4.0) license, which permits others to distribute, remix, adapt, build upon this work non-commercially, and license their derivative works on different terms, provided the original work is properly cited and the use is non-commercial. See: http://creativecommons.org/ licenses/by-nc/4.0/

(C) Article author(s) (or their employer(s) unless otherwise stated in the text of the article) 2017. All rights reserved. No commercial use is permitted unless otherwise expressly granted.

\section{REFERENCES}

1. Leiria TL, Pellanda LC, Magalhães E, et al. Comparative study of a portable system for prothrombin monitoring using capillary blood against venous blood measurements in patients using oral anticoagulants: correlation and concordance. Arq Bras Cardiol 2007;89:1-5.

2. Yeh $\mathrm{CH}$, Hogg K, Weitz Jl. Overview of the new oral anticoagulants: opportunities and challenges. Arterioscler Thromb Vasc Biol 2015;35:1056-65.

3. Madrid C, Sanz M. What influence do anticoagulants have on oral implant therapy? A systematic review. Clin Oral Implants Res 2009;20:96-106.

4. Dahlbäck B. Blood coagulation. Lancet 2000;355:1627-32.

5. Harter K, Levine M, Henderson SO. Anticoagulation drug therapy: a review. West J Emerg Med 2015;16:11-17.

6. King N, Tran MH. Long-acting anticoagulant rodenticide (Superwarfarin) poisoning: a review of its historical development, epidemiology, and clinical management. Transfus Med Rev 2015;29:250-8.

7. Thachil J, Gagg J. Problem-based review: non Vitamin K Antagonist Oral Anticoagulants for the Acute Physician. Acute Med 2015;14:83-9.

8. Campbell JH, Alvarado F, Murray RA. Anticoagulation and minor oral surgery: should the anticoagulation regimen be altered? $\mathrm{J}$ Oral Maxillofac Surg 2000;58:131-5.

9. Vicente Barrero M, Knezevic M, Tapia Martín M, et al. Oral surgery in patients undergoing oral anticoagulant therapy. Med Oral 2002;7:63-70.

10. Evans IL, Sayers MS, Gibbons AJ, et al. Can warfarin be continued during dental extraction? Results of a randomized controlled trial. $\mathrm{Br}$ J Oral Maxillofac Surg 2002;40:248-52.

11. Sacco R, Sacco M, Carpenedo M, et al. Oral surgery in patients on oral anticoagulant therapy: a randomized comparison of different intensity targets. Oral Surg Oral Med Oral Pathol Oral Radiol Endod 2007;104:e18-21.

12. Al-Mubarak S, Al-Ali N, Abou-Rass M, et al. Evaluation of dental extractions, suturing and INR on postoperative bleeding of patients maintained on oral anticoagulant therapy. Br Dent J 2007;203:1-5.

13. Morimoto $\mathrm{Y}$, Niwa $\mathrm{H}$, Minematsu K. Hemostatic management of tooth extractions in patients on oral antithrombotic therapy. $J$ Oral Maxillofac Surg 2008;66:51-7.

14. Aframian DJ, Lalla RV, Peterson DE. Management of dental patients taking common hemostasis-altering medications. Oral Surg Oral Med Oral Pathol Oral Radiol Endod 2007;103(Suppl 1):S45.e1-11.

15. Goodchild JH, Donaldson M. An evidence-based dentistry challenge: treating patients on warfarin (Coumadin). Dent Implantol Update 2009;20:1-8

16. Nematullah A, Alabousi A, Blanas N, et al. Dental surgery for patients on anticoagulant therapy with warfarin: a systematic review and meta-analysis. J Can Dent Assoc 2009;75:41.
17. Bajkin BV, Vujkov SB, Milekic BR, et al. Risk factors for bleeding after oral surgery in patients who continued using oral anticoagulant therapy. J Am Dent Assoc 2015;146:375-81.

18. Iwabuchi $\mathrm{H}$, Imai $\mathrm{Y}$, Asanami $\mathrm{S}$, et al. Evaluation of postextraction bleeding incidence to compare patients receiving and not receiving warfarin therapy: a cross-sectional, multicentre, observational study. BMJ Open 2014:4:e005777.

19. van Diermen DE, van der Waal I, Hoogstraten J. Management recommendations for invasive dental treatment in patients using oral antithrombotic medication, including novel oral anticoagulants. Oral Surg Oral Med Oral Pathol Oral Radiol 2013;116:709-16.

20. Yang S, Shi Q, Liu J, et al. Should oral anticoagulant therapy be continued during dental extraction? A meta-analysis. BMC Oral Health 2016;16:81.

21. Shi Q, Xu J, Zhang T, et al. Post-operative Bleeding Risk in Dental Surgery for Patients on Oral Anticoagulant Therapy: A Meta-analysis of Observational Studies. Front Pharmacol 2017;8:58.

22. Kämmerer PW, Frerich B, Liese J, et al. Oral surgery during therapy with anticoagulants-a systematic review. Clin Oral Investig 2015;19:171-80.

23. Higgins JPT, Green S. Cochrane Handbook for Systematic Reviews of Interventions Version 5.1.0: The Cochrane Collaboration, 2011. www.cochrane-handbook.org.

24. Cochrane. Trusted evidence.Informed decisions.Better health. 2014 http://www.cochrane.org/training/ CHfSRolTCC, 2014) c-hAJ.

25. Moher D, Liberati A, Tetzlaff J, et al. Preferred reporting items for systematic reviews and meta-analyses: the PRISMA statement. Ann Intern Med 2009;151:264-409.

26. Holbrook A, Schulman S, Witt DM, et al. Evidence-based management of anticoagulant therapy: Antithrombotic Therapy and Prevention of Thrombosis, 9th ed: American College of Chest Physicians Evidence-Based Clinical Practice Guidelines. Chest 2012;141:e152S-84.

27. Guyatt GH, Oxman AD, Kunz R, et al. GRADE guidelines: 7 . Rating the quality of evidence--inconsistency. J Clin Epidemiol 2011;64:1294-302.

28. Altman R, Alarcón G, Appelrouth D, et al. The American College of Rheumatology criteria for the classification and reporting of osteoarthritis of the hand. Arthritis Rheum 1990;33:1601-10.

29. AkI EA, Johnston BC, Alonso-Coello P, et al. Addressing dichotomous data for participants excluded from trial analysis: a guide for systematic reviewers. PLoS One 2013;8:e57132.

30. Oxman AD, Cook DJ, Guyatt GH. Users' guides to the medical literature. VI. How to use an overview. Evidence-Based Medicine Working Group. JAMA 1994;272:1367-71.

31. Guyatt GH, Oxman AD, Montori V, et al. GRADE guidelines: 5. Rating the quality of evidence--publication bias. J Clin Epidemiol 2011;64:1277-82.

32. Higgins JP, Thompson SG. Quantifying heterogeneity in a metaanalysis. Stat Med 2002;21:1539-58.

33. Busse JW, Bartlett SJ, Dougados M, et al. Optimal Strategies for Reporting Pain in Clinical Trials and Systematic Reviews: Recommendations from an OMERACT 12 Workshop. J Rheumatol 2015;42:1962-70.

34. Johnston BC, Thorlund K, Schünemann $\mathrm{HJ}$, et al. Improving the interpretation of quality of life evidence in meta-analyses: the application of minimal important difference units. Health Qual Life Outcomes 2010;8:116.

35. Suissa S. Binary methods for continuous outcomes: a parametric alternative. J Clin Epidemiol 1991;44:241-8.

36. Thorlund K, Walter SD, Johnston BC, et al. Pooling health-related quality of life outcomes in meta-analysis-a tutorial and review of methods for enhancing interpretability. Res Synth Methods 2011:2:188-203.

37. Ebrahim S, Akl EA, Mustafa RA, et al. Addressing continuous data for participants excluded from trial analysis: a guide for systematic reviewers. J Clin Epidemiol 2013;66:1014-21.

38. Rennie D, Meade M, Cook D, eds. Users' guides to the medical literature: a manual for evidence-based clinical practice: McGraw-Hill, 2008.

39. Orwin RG. Evaluating coding decisions. In: Cooper H, Hedges LV, eds. The handbook of research synthesis. New York, NY: Russell Sage Foundation, 1994:555-62.

40. Guyatt GH, Thorlund K, Oxman AD, et al. GRADE guidelines: 13. Preparing summary of findings tables and evidence profilescontinuous outcomes. J Clin Epidemiol 2013;66:173-83.

41. Guyatt $\mathrm{GH}$, Oxman AD, Kunz R, et al. Going from evidence to recommendations. BMJ 2008;336:1049-51. 